\title{
Holistic Service Orchestration over Distributed Micro Data Center
}

\author{
TaeYeon Kim ${ }^{1,2}$ and Hongseok Jeon ${ }^{1,2}$ \\ ${ }^{1}$ Network Computing Convergence Research Section, Smart Network Research \\ Department, Korea \\ ${ }^{2}$ Electronics and Telecommunications Research Institute (ETRI), Korea \\ E-mail: $\{$ tykim; jeonhs\}@etri.re.kr
}

Received 15 November 2016; Accepted 7 December 2016;

Publication 24 December 2016

\begin{abstract}
Three technologies, i.e. Cloud, SDN, and NFV, the most frequently touted as promising terms to pave the way to the next generation networking era are keenly needed to be harmonized for vertical application service providers. This holistic approach to orchestrate application service on the edge of the network is based on requirements such as Service Profile and Service Policy with environmental condition from service providers and network providers respectively. This paper shows how to organize and manage the application service from the perspective of end user side with two requirements, profile and policy, leveraging three disruptive technologies.
\end{abstract}

Keywords: Cloud, SD, NFV, orchestration.

\section{Introduction}

Cloud networks provide various services on top of virtualized compute and storage resources. The flexible operation and optimal usage of the underlying infrastructure are realized by resource orchestration methods and virtualization techniques developed during the recent years [1].

Also, networking and software design principles converge currently under the notions of Software-Defined Networking (SDN) and Network Function

Journal of Software Networking, 23-34.

doi: 10.13052/jsn2445-9739.2017.002

(C) 2017 River Publishers. All rights reserved. 
Virtualization (NFV). This means that network services are not anymore static and manually configured, but they become flexible and in the end virtualized components. This convergence enables to dynamically orchestrate the network, to move network functions to the cloud and to direct and prioritize traffic intelligently. Applying cloud principles to network services, configuration and management requires mechanisms for automation, virtualization and elasticity. Ultimately, network services may improve the customer experience for the end users requiring on-demand network resources [2].

Cloud management technology enables computing environment including server, storage and networking between them. Virtual networking functions for the service will be deployed and managed by NFV technology and packets from the customers of the service will be directed and followed along the designated path which is established by the packet forwarding policy making use of SDN technology [3, 4].

This paper suggests holistic service orchestration methodology and procedures accommodating service features based on demand from the vertical service providers by leveraging cloud, SDN and NFV capabilities as well as adaptive service management techniques along with environmental variables. In Section 2, holistic orchestration concept and its features will be described in more detail. Case study of the process are covered in Section 3 followed by conclusion in Section 4.

\section{Orchestration over Distributed Cloud-NFV Platform}

A distributed Cloud-NFV platform refers to an open platform for realization of network functions virtualization based on cloud and software defined networking technologies. It includes a global service manager (GSM) and at least one Micro Data Center (MDC) as shown in Figure 1.

The GSM integrally controls and manages cloud resources, i.e. MDCs in the distributed cloud environment. The GSM may provide a Service Profile input from the service provider and a service according to a Service Policy information to the service user. Further, the GSM orchestrates the service according to various environment factors which may be changed during execution of the service.

The MDC includes at least one virtual functions and the application servers. For example, the virtual functions and the application servers of one MDC are virtually linked with a virtual functions and an application servers of other MDC and an application servers of another MDC, and may provide a service according to a service flow to the service end user. 


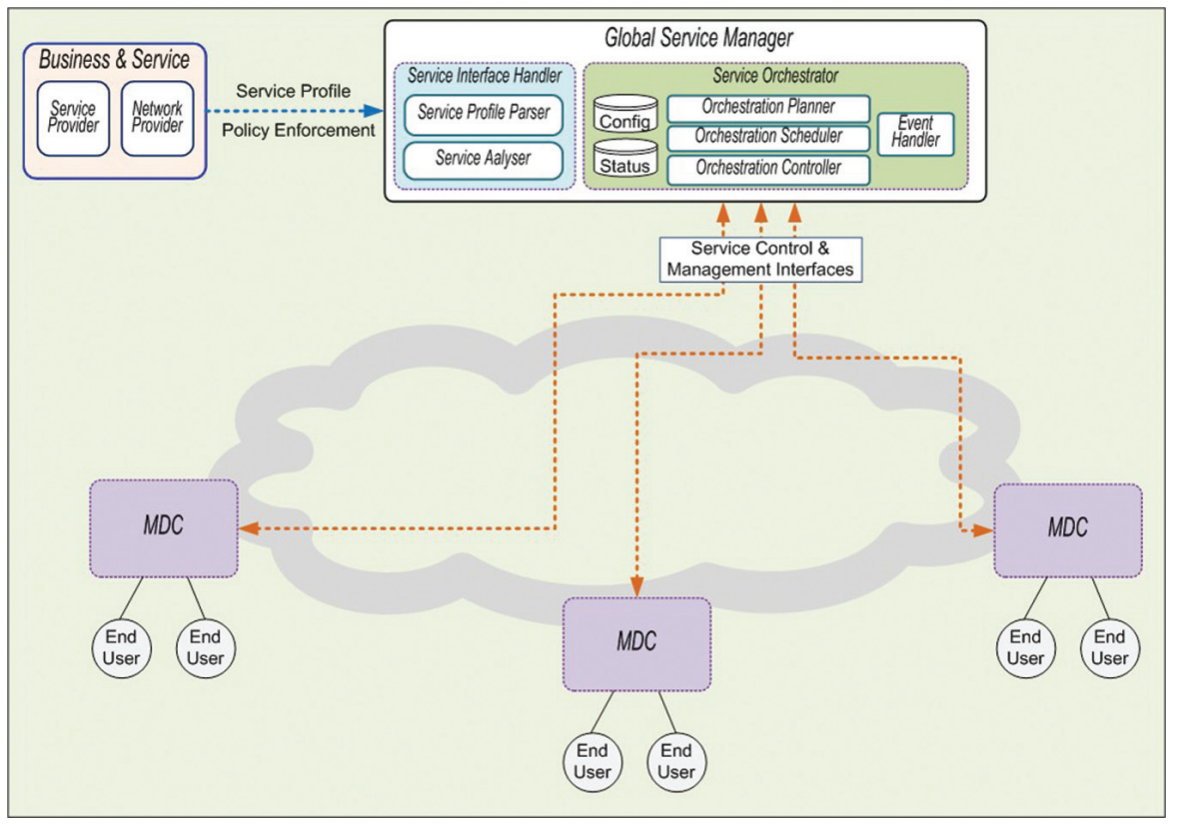

Figure 1 A logical framework of distributed cloud-NFV platform.

\subsection{Service Interface Handler}

A GSM includes a Service Interface Handler and a Service Orchestrator. The Service Interface Hander has a Service Profile Parser and a Service Analyzer in it. The Service Interface Handler may receive the process a Service Profile and service providing policies from the service provider. The Service Specification may be generated as a result of the processing.

The Service Profile Parser may receive the Service Profile and the service providing policy. The Service Profile includes overall information necessary for providing the service such as contents of the service to be provided the service such as contents of the service to be provided from the service provider, information on operational control of the MDC and control information on execution, change, stop, and restart of the service. The Service Profile may be configured by abstractive natural languages. The service providing policy is a factor which causes influence upon the service flow and may be received from the network provider and service provider.

The Service Profile Parser may classify the service indicated by the Service Profile according to attribute by parsing the Service Profile input 
from the service provider. In detail, the Service Profile Parser may classify attributed of the service according to the information included in the Service Profile. By going through Service Analyzer, service attribute information and environment condition information for providing the service may be obtained. In this case, the Service Policy information may be considered as one of the environment condition information to be considered when the service is initially provided or when the service is changed. Operation of the service to be offered may be changed according to the environment condition information such as:

- Status condition: operation may be changed according to status of the network, MDC and the virtual functions

- Time slot condition: server operation may be changed according to time slot

- Service level agreement condition: service operation may be changed based on SLA

- Zone condition: service operation may be changed according to a service request zone

- Service flow condition: service operation may be changed according to the service flow

- Service providing policy: service attribute detail information may be changed according to a corresponding service providing policy when a certain policy is proposed and transmitted by the service provider or the service manager. Service scaling, service mobility, and service chaining policy may be changed according to the service providing policy.

\subsection{Service Orchestrator}

The Service Orchestrator includes an Orchestration Planner, an Orchestration Controller and an Event Handler as illustrated in Figure 2.

The Orchestration Planner analyzes the Service Specification transferred from the Service Interface Handler and recognize the capacity and the function of the MDC required by the service attribute detail information of the Service Specification. The Orchestration Planner may specify MDC candidates satisfying the function and the processing capacity of the MDC required by the Service Specification with reference to the configuration information.

At operation, the Orchestration Planner may decide an optimal MDC from the MDC candidates with reference to the status information. In order to decide the optimal MDC, service attribute, regional characteristic, and 


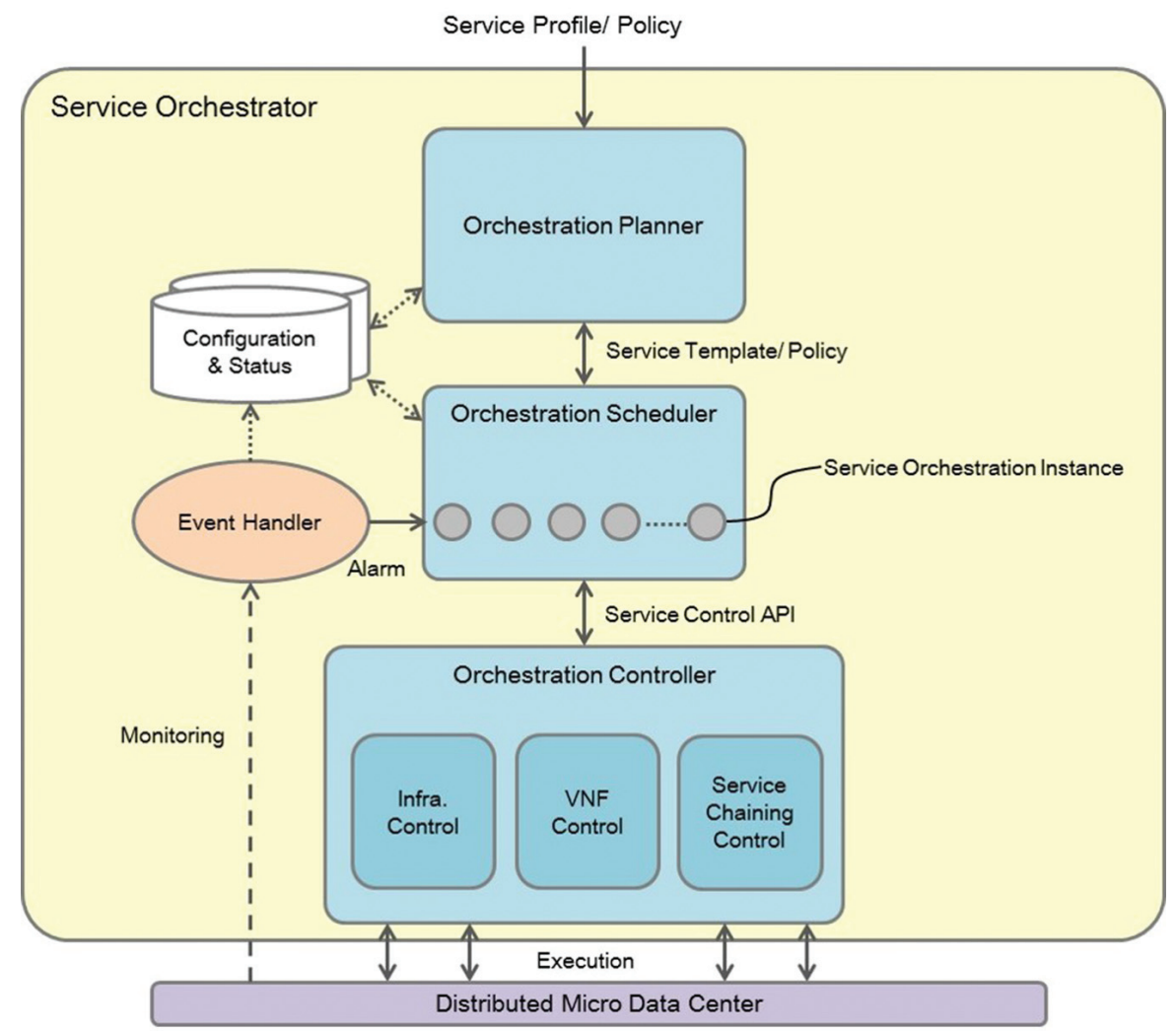

Figure 2 Functional architecture of service orchestrator.

host environment may be considered. The Orchestration Planner determines whether an optimal MDC is decided with respect to all MDCs required by the service attribute information of the Service Specification.

As a next step, the Orchestration Planner identifies virtual functions and the application servers required for the MDC to be deployed the service. The virtual functions and the application servers may be configured to be mapped to the MDC and to be operated as an independent functional entity in a virtual network which is composed of multiple virtual links between virtual entities. Though the connection of the virtual link, a virtual network considering the attribute of the service, the regional characteristics and the host environment may be configured. The Orchestration Planner may generate a Service Template based on the virtual network configuration information 
generated together with the attribute information and the condition information include in the Service Specification. The Orchestration Planner provides the Service Template to the Orchestration Scheduler which is supposed to create service orchestration instances based on the Service Template received from the Orchestration Planner.

The Orchestration Scheduler generates the service instance messages such as initialization and changes according to the Service Orchestration Instance and transfer the generated service instance message to the Orchestration Controller. The service instance message includes information on the service flow and information on associated virtual functions and application servers.

For example, the Orchestration Scheduler may firstly generate the Service Orchestration Instance by selecting the virtual functions and the application servers necessary for initial service execution according to the generated Service Orchestration Instance. Secondly, it decides the service flow with respect to the selected virtual functions and application servers for initial service execution and information on the relevant MDC and virtual functions included in the initial execution instance message.

Meanwhile, the Orchestration Scheduler may register the environment condition information included in the Service Template to the Event Handler. When the Orchestration Scheduler receives an event indication that meets registered environment condition information from the Event Handler, the Orchestration Scheduler selects the virtual functions and the application servers necessary for the execution of the service change, and connect the virtual functions with the application servers with reference to the service attribute detail information. The service flow for the execution of the service change and information on the relevant virtual functions and application servers may be included in a change execution instance message.

The Orchestration Controller may control the virtual functions and the application servers distributed in the MDC based on the received service instance message. In detail the service or controller may transfer a service execution control command through an interface engaged with the distributed MDC. Accordingly the service according to the service flow may be provided to the user. When the Orchestration Controller receives the initial execution instance message the controller obtains the service flow of the service to be firstly provided on the virtual network according to the initial execution instance message. The Orchestration Controller may transmit a service execution control command for the virtual functions and application servers of the MDC. 


\section{Case Study of Holistic Service Orchestration}

As an example, media service which is supposed to provide real-time or ondemand video images to the end users coming through the cameras and video servers spread around the multiple region. This type of service is required to offer synthesized images coming from multiple origins or deliver stored videos based on demand.

For the sake of efficiency of the storage, only images of moving objects are stored and processed for offering to the end user when those images are requested. Some images will be cached in the local area in case of public content. Service may provide local-customized application conveying commercials from the local area of the end users with the content that user requested.

\subsection{Service Profile}

As the first stage of the service deployment, Service Profile registered via service platform interface will be parsed and analyzed to produce Service Specification which contains service characteristics such as a following example.

- Service identifier: Unique identifier of the service to be deployed

- Scope of Service (Local or Global): Global

- Type of Service (application type or networking type): application type

- Application servers Information (required application servers and attributes): image synthesizer, media, storage, range of the number of servers, performance indicators

- Virtual Network Functions Information (required VNFs and attributes): image filter, virtual switch, transcoder, cache, advertiser, load balancer with their performance, the number of instances of the VNFs

- Performance (Requirement from the perspective of end users, latency, bandwidth, frequency of use) lower than $100 \mathrm{~ms}$ latency, higher than 20 Mbps, 5 times, 2 hours/day

- Scaling policy (Scaling condition of application servers and VNFs, applicable for scale up/down, scale in/out): scale out in case of more than $2 \mathrm{k}$ session per server

- Mobility policy (Server migration condition): 2 hours pertaining service with over $100 \mathrm{~ms}$ latency

- Chaining policy (multiple VNFs and value added function chaining condition): acceleration depending on SLA, VNFs chaining based on time zone and traffic volume. 


\subsection{Service Orchestration Process}

Figure 3(a) shows a Service Specification for virtualized media service specifying required application servers, virtual network functions which are identified and extracted from the Service Profile provided by the media service provider. The Service Specification produced from a Service Profile will be transferred to Service Planner of the Service Orchestration System to develop the Service Template by comparisons of current resource capacity and status with service requirements specified on the Service Specification. Optimized selection of the region for placement of application servers and virtual network functions will be performed for service provisioning with virtual links identified for the establishment of networking environment between servers and VNFs.

A Service Template in Figure 3(b) denotes provisioning model distributing application servers and VNFs over multiple MDCs with optimization policy based on information specified on the Service Specification such as frequency of use and performance metrics of the service components. Application servers and VNFs deployed in distribution would be connected through virtual links with layer 2 connectivity for building a virtual network between them which is distinguished from the physical connection. After all, Service Template process in the Orchestration Planner means the construction of service infrastructure by displaying server and function with network between them.

Orchestration Scheduler performs establishment of the service forwarding path, i.e. Service Instance on the Service Template by creation of a Service

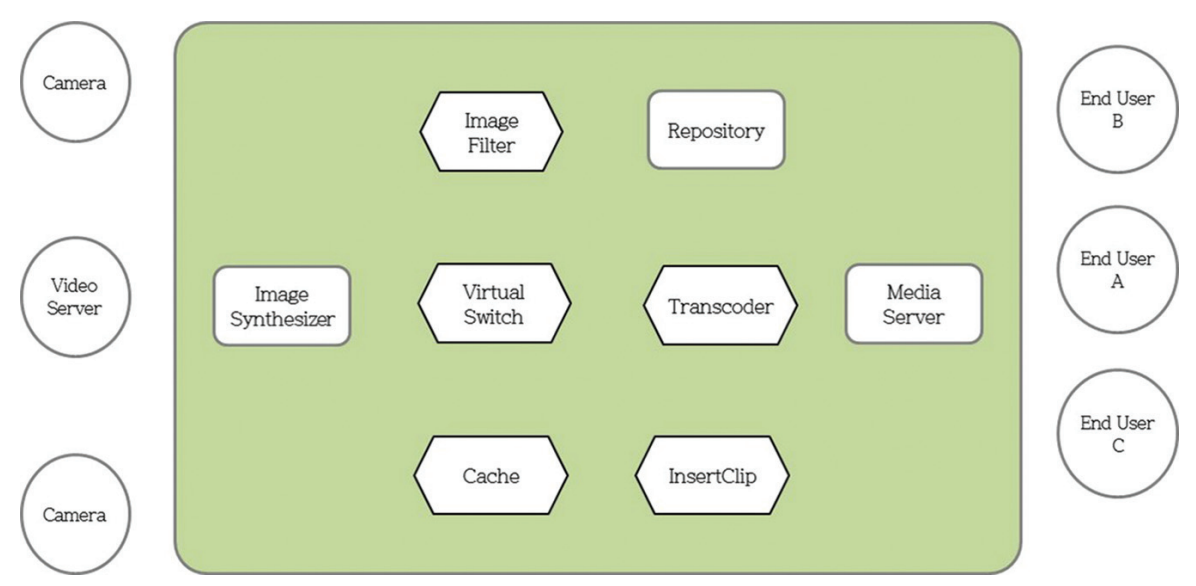

(a) Service Specification 


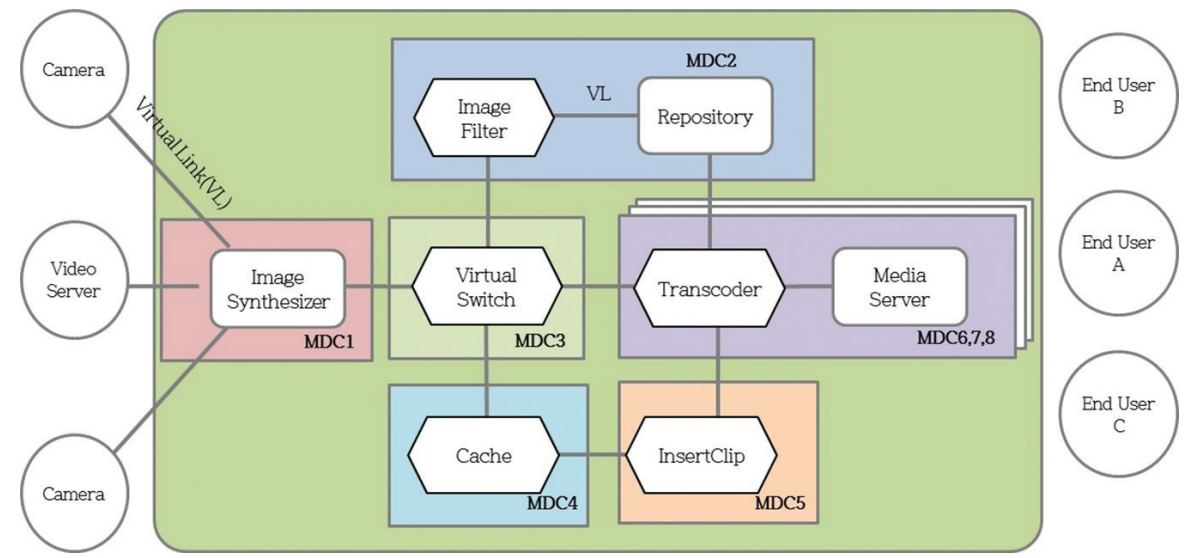

(b) Service Template

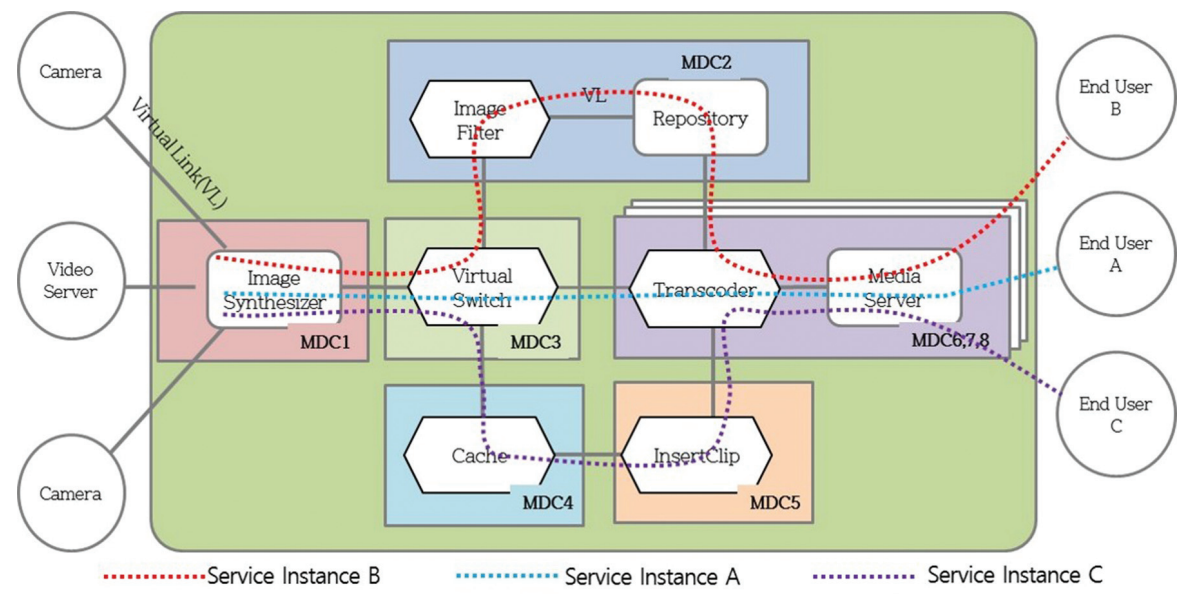

(c) Service Instance

Figure 3 An example of service orchestaration process.

Orchestration Instance which manages corresponding Service Instance. The Service Orchestration Instance performs service instance management through the APIs of MDC.

An initial service instance incorporates servers, functions and virtual links into a service forwarding path in early stage. For the end user A, in the Figure 3(c), initial service which synthesize images out of video content and cameras will be instantiated for the initial service instance. To this end, traffic 
flow out of the image server will be classified through virtual switch and traverse transcoder and media server before it arrived to the end user. The initial service of the example requires two application servers, synthesizer and media server, and two virtual functions, virtual switch and transcoder respectively. After the creation of a Service Instance, Orchestration Scheduler takes its control over Orchestration Controller which provides interface to execute the Service Instance. Infrastructure Controller has the responsibility of execution of creation, destruction, modification of virtual resource related to application servers and virtual networks. Virtual functions Controller takes roles of management and control for the virtual functions. Chaining control between VNFs are controlled and managed by Service Chaining Controller respectively.

For an example of Orchestration Controller operation, we suppose End User B wants to have a new video service keeping record of motion images only. To meet the service requirements two additional functions are identified and incorporated in the service by changing packet forwarding path through Service Chaining Controller to create a new Service Instance which traverse newly-added two functions, image-filter function and repository function. The same process is true with the creation of another Service Instance for end user $\mathrm{C}$ which is the applicable for the instant commercial insertion application service allowing real-time video to traverse Cache and InsertClip functions by adding a new Service Instance C into the Service Template

\section{Conclusion}

Network function virtualization (NFV) and software defined networking (SDN) are key technology enablers for cost reductions and new business models in networking [5]. This paper shows a stepwise service orchestration procedures taking possible variables into account such as service profile, policies, environmental conditions and their variations for application oriented service deployment over distributed micro data centers. By leveraging Cloud, SDN and NFV technologies the programmable infrastructure will pave the way to the forthcoming ICT era for application and service-tailored infrastructure. Especially management schemes for scaling, mobility and chaining in the scope of virtual environment should be investigated from the holistic view from the end users perspectives. 


\section{References}

[1] Sonkoly, B., Szabo, R., Jocha, D., Czentye, J., Kind, M., Westphal, F.-J. (2015). "UNIFYing Cloud and Carrier Network Resources: An Architectural View," in IEEE GLOBECOM Global Communications Conference (GLOBECOM) (Rome: IEEE), 1-7. doi: 10.1109/GLOCOM. 2015.7417869

[2] Carella, G., Yamada, J., Blum, N., Lck, C., Kanamaru, N., Uchida, N., Magedanz, T. (2015). "Cross-layer service to network orchestration," 2015 IEEE International Conference on Communications (ICC) (Rome: IEEE). doi: 10.1109/ICC.2015.7249414

[3] Kim, T., and Lee, B. (2014). "Scalable CDN Service PoC over Distributed Cloud Management Platform," in 2014 International Conference on Information and Communication Technology Convergence (ICTC) (IEEE: Rome). doi: 10.1109/ICTC.2014.6983304

[4] Kim, T., and Lee, B. (2015). "State-Driven Service Orchestration for Transparent Internet Caching," in 2015 International Conference on Information and Communication Technology Convergence (ICTC) (IEEE: Rome). doi: 10.1109/ICTC.2015.7354654

[5] Szabo, R., Kind, M., Westphal, F.-J., Woesner, H., Jocha, D., Csaszar, (2015). Elastic network functions: opportunities and challenges. IEEE Netw. 29, 15-21. doi: 10.1109/MNET.2015.7113220

\section{Biographies}

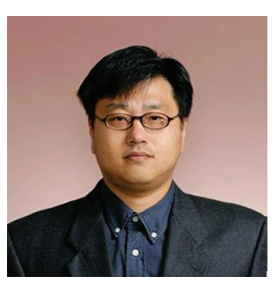

T. Kim received Ph.D. degree in computer science from ChungBuk National University, Korea, in 2007. He also received B.S. and M.S. degrees from Chung-Ang University, Seoul, in 1990 and 1992, respectively. He joined Electronics and Telecommunications Research Institute (ETRI) in 1992. His research includes network \& computing convergence platform, SDN and NFV technologies for future network. 


\section{T. Kim and H. Jeon}

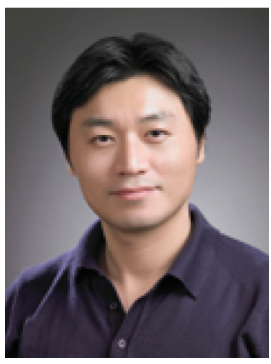

H. Jeon received the B.S. degree in Industrial Engineering from Sungkyunkwan University, Seoul, Korea, in 2002. He received the M.S. degree in Engineering from Information and Communications University (ICU), Daejon, Korea, in 2004. He joined Electronics and Telecommunications Research Institute (ETRI) in 2004. He is interested in the SoftwareDefined Networking (SDN) and Network Functions Virtualization (NFV). 